Research Paper

\title{
Olfactory stimulation modulates the blood glucose level in rats
}

\author{
Tadataka Tsuji ${ }^{\bowtie}$, Susumu Tanaka ${ }^{1}$, Sanam Bakhshishayan ${ }^{1}$, Mikihiko Kogo ${ }^{1}$ and Takashi Yamamoto ${ }^{2}$ \\ 1. The First Department of Oral and Maxillofacial Surgery, Graduate School of Dentistry, Osaka University, Osaka 565-0871, Japan. \\ 2. Department of Health and Nutrition, Faculty of Health Science, Kio University, Nara 635-0832, Japan. \\ $\triangle$ Corresponding author: Tadataka Tsuji, DDS, Ph D. The First Department of Oral and Maxillofacial Surgery, Graduate school of Dentistry, Osaka University, \\ 1-8 Yamadaoka, Suita City, Osaka, 565-0871, Japan. Tel: +81-6-6879-2936, Fax: +81-6-6876-5298, E-mail: g2787b@dent.osaka-u.ac.jp \\ (C) Ivyspring International Publisher. This is an open access article distributed under the terms of the Creative Commons Attribution (CC BY-NC) license \\ (https://creativecommons.org/licenses/by-nc/4.0/). See http://ivyspring.com/terms for full terms and conditions.
}

Received: 2017.06.19; Accepted: 2018.01.12; Published: 2018.01.18

\begin{abstract}
In both humans and animals, chemosensory stimuli, including odors and tastes, induce a variety of physiologic and mental responses related to energy homeostasis, such as glucose kinetics. The present study examined the importance of olfactory function in glucose kinetics following ingestion behavior in a simplified experimental scenario. We applied a conventional glucose tolerance test to rats with and without olfactory function and analyzed subsequent blood glucose (BG) curves in detail. The loss of olfactory input due to experimental damage to the olfactory mucosa induced a marked decrease in the area under the BG curve. Exposure to grapefruit odor and its main component, limonene, both of which activate the sympathetic nerves, before glucose loading also greatly depressed the BG curve. Pre-loading exposure to lavender odor, a parasympathetic activator, stabilized the BG level. These results suggest that olfactory function is important for proper glucose kinetics after glucose intake and that certain fragrances could be utilized as tools for controlling BG levels.
\end{abstract}

Key words: blood glucose, odor, glucose tolerance test, the area under the curve of blood glucose

\section{Introduction}

Certain food ingredients, such as carbohydrates, are known to raise blood glucose (BG) levels, which are generally controlled by internal mechanisms of energy homeostasis. Glucose absorption from the gastrointestinal (GI) tract and GI motility are meditated by the autonomic nervous system (ANS), suggesting that the ANS plays an important role in regulating BG levels via a neurohumoral mechanism [1].

Feeding-related sensory stimuli, such as the color, odor, taste, tenderness, and sound of foods, play important roles not only in constructing food preferences and triggering the desire to eat [2] but also in modulating physiologic functions. For example, sweet tastes induce the cephalic phase of insulin release [3] via the vagus nerve [4] and increased gastric emptying [5], suggesting the possibility of modulating BG levels via the ANS. Odor stimulation also influences the ANS. For example, the scent of grapefruit and its main component, limonene, increases and decreases the activity of sympathetic and parasympathetic nerves, respectively [6]. The chemical senses, such as olfaction and taste, could be important factors in modulating the BG level during ingestion behavior through the action of the ANS. However, to date, there are no reports of studies examining the direct action of these chemosensory stimuli on the hyperglycemic response.

In a previous study, we reported the effect of depriving one of the chemical senses on glucose kinetics in humans [7]. Direct intragastric delivery of glucose or clamping the nose closed during glucose intake induced a downward shift in both the BG and serum insulin response (IR) curves, resulting in a decrease in the area under the BG curve, which was positively correlated with the area under the IR curve. To verify and further extend the previous findings, in the present study, we examined how blocking odors 
before and during ingestion of glucose affects BG in rats. We discuss whether chemosensory stimulation, especially by odors, is a suitable strategy for modulating ingestion-induced changes in BG levels.

\section{Materials and Methods}

\section{Animals}

Adult male Wistar rats $(n=23$, weighing 250-300 g) were obtained from the Charles River Breeding Laboratories (Kanagawa, Japan). Rats were housed separately in a controlled-environment room $\left(23^{\circ} \mathrm{C}\right.$, $60 \%$ humidity, 12-h light: 12 -h dark cycle) and given ad libitum access to food (MF Pellets; Oriental Yeast, Tokyo, Japan) and water. All experiments were carried out in accordance with the Guidelines laid down by the NIH in the US regarding the care and use of animals for experimental procedures, and were approved by the Institution Animal Care and Use Committee of Osaka University.

\section{Anosmic treatment}

The rats were trained to perform a cookie-finding task designed to evaluate olfactory capacity. After being deprived of food for $15 \mathrm{~h}$, each rat was placed in a plastic chamber $(400 \mathrm{~mm} \times 270$ $\mathrm{mm} \times 200 \mathrm{~mm}$ ) and the floor of the apparatus was covered with approximately $(7 \mathrm{~cm})$ of woodchip (white flake; Oriental Yeast, Tokyo, Japan). An Oreo cookie (diameter, $4.5 \mathrm{~cm}$; Nabisco, Japan), with which the rats were previously familiarized with, was placed in a plastic cage until the rats consistently ate them. Subsequently, sawdust was poured on top of the cookie and spread in the cage to a depth of $3 \mathrm{~cm}$. After placing a rat in the middle of the cage, the period of latency prior to finding the cookie was measured. Each rat was removed from the chamber either after the complete consumption of the cookie or at the end of the 3 min period.

Once all of the rats found the cookie in less than 3 min (Fig. 1A), they were anesthetized with halothane and placed on their backs on an inclined surface, with their heads facing downward. The zinc sulfate $\left(\mathrm{ZnSO}_{4}\right)$ solution $(10 \%$ [wt/vol] in saline) was delivered to the bilateral nasal cavity via a 26-gauge blunted needle until $0.5 \mathrm{ml}$ drained out of the external nares (anosmia groups, $n=6$ ) [8]. Sham-treated rats (control groups, $n=6$ ) were anesthetized and nasally perfused with saline instead of $\mathrm{ZnSO}_{4}$. Post-treatment, all rats were housed individually in plastic cages. The next morning, a cookie-finding task was performed to confirm that the rats treated with $\mathrm{ZnSO}_{4}$ were anosmic. $\mathrm{ZnSO}_{4}$-treated rats that could not actively find the cookie within 9 min were considered anosmic and included in the study.

\section{Olfactory stimuli}

The essential oils of grapefruit, lavender, and limonene (supplied by R. Komaki at the Seisyo Aroma Institute, Kanagawa, Japan) were used as olfactory stimuli in Experiment 2. For olfactory stimulation, a piece of chromatography paper $(5 \mathrm{~mm} \times 25 \mathrm{~mm}$ ) was soaked in $20 \mu \mathrm{l}$ of essential oil solution and placed on the floor of a plastic chamber $(400 \mathrm{~mm} \times 270 \mathrm{~mm} \times 200$ $\mathrm{mm})$, the top of which was then covered to preserve the odor. After each trial, the chamber was cleaned with a combination of bleach/water and then rinsed with water to permit evaporation of any residual odor. We placed the scented paper under the nose of each rat in a holder such that the animal would continuously sniff the odor during breathing for 15 min. The rats were then transported to the new chamber after a period of removal from the odor, and the BG level was then continuously monitored.

\section{Experimental protocol}

BG levels were measured in rats subjected to a conventional glucose tolerance test after being deprived of food for $15 \mathrm{~h}$. The procedure was performed on the same rats under the following conditions on different days in each study. Before the day of the experiment, each rat was trained to quietly remain in a holder (Natsume Seisakusho Co., Ltd. Osaka, Japan) for several hours and continuously drink glucose solution through the front of the nasal cone from a dropper.

All experiments were begun at 9:00 $\mathrm{AM}$ and finished by noon to minimize the effects of diurnal rhythm. First, we placed the rat in a holder with adjustable front nose cone to limit turning and movement. The rat's nose protrudes through the front of the nose cone, allowing for comfortable breathing. Next, we fed the rat $20 \%$ glucose solution (glucose/body weight $=1 \mathrm{mg} / \mathrm{g}$, glucose-loading) for $3 \mathrm{~min}$. The rat's tail should be fully extended and exit through the rear hatch opening of the holder, allowing for blood sampling from the tail. Then blood samples were collected at fixed time intervals: $-3,15$, $30,45,60,90$, and $120 \mathrm{~min}$ after glucose loading. Blood was collected from the tail vein using a 26-gauge needle and syringe. The BG level was measured at least twice to minimize the effects of random errors.

\section{Experimental design}

Experiment 1: Effect of impaired olfaction on BG after glucose loading

We performed the oral glucose tolerance test before and after anosmic treatment in anosmic groups to determine the effect of impaired olfaction on BG after glucose loading. We compared the BG curves for 
2 scenarios: before or after anosmic treatment $(n=6)$.

Experiment 2: Effect of addition of olfactory stimulus on BG after glucose loading

\section{Experiment 2-1}

We examined the effect of a pre-glucose loading olfactory stimulus on the BG curve using two odors, grapefruit and lavender, as possible counterparts to the ANS [6,9], which normally controls the digestive system. We conducted an oral glucose tolerance test after a 15-min period of sniffing grapefruit or lavender odor, in comparison with control rats exposed to odor-free air in same rats $(n=6)$.

\section{Experiment 2-2}

We selected limonene, which constitutes 93.3\% of grapefruit oil [6], as the active component for examining the effect of grapefruit odor on BG. An oral glucose tolerance test was performed after sniffing grapefruit or limonene for $15 \mathrm{~min}(\mathrm{n}=5)$.

\section{Experiment 2-3}

We investigated the effect of grapefruit odor on BG level in anosmic rats to determine whether the effect is induced by (i) olfactory neural information transmitted from the olfactory cells to the brain or (ii) humoral transmission of the odor molecules absorbed from the respiratory mucosa to the brain. We then compared the BG curves of rats treated with either grapefruit odor or odor-free air $(n=6)$.

\section{AUBGC}

We calculated the area under the BG curve (AUBGC) by using the trapezoidal rule with baseline as the $x$-axis [7]. Thus the AUBGC is used to analyze the BG curve, in which the area is described as:

$$
\mathrm{AUC} \fallingdotseq\left(\mathrm{Y}_{-3}+\mathrm{Y}_{15}\right) \times(3+15) / 2+\left(\mathrm{Y}_{15}+\mathrm{Y}_{30} \times 2+\mathrm{Y}_{45} \times\right.
$$
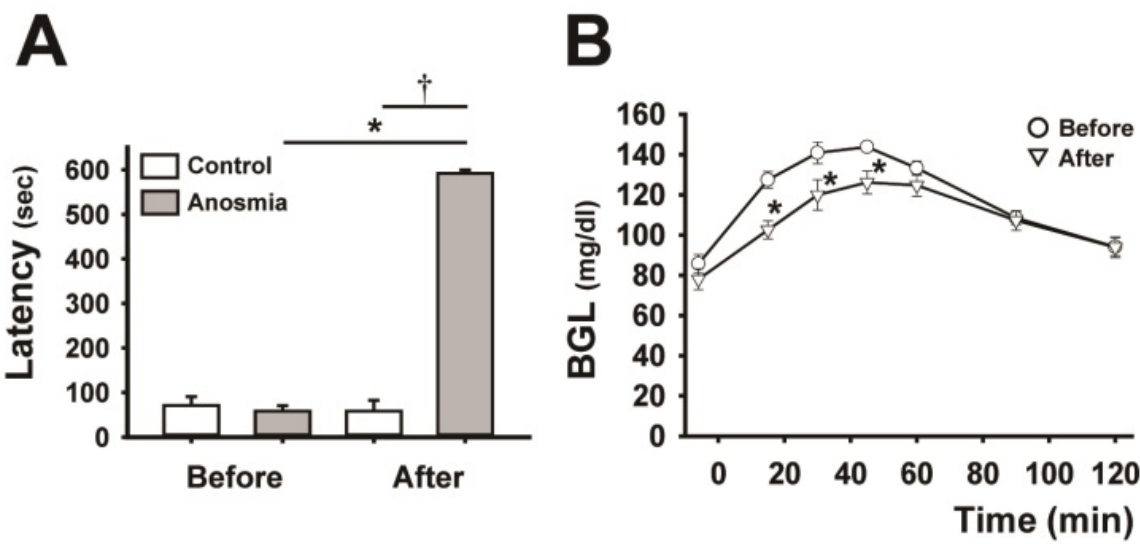

Figure 1. Effect of anosmic treatment on the blood glucose curve. A, comparison of the latency to the finding of the cookie before and after the anosmic treatment in the control and anosmic groups. Data are mean \pm SEM ( $n=6$, per each group), $* P<0.05$ (after vs. before anosmic treatment), Wilcoxon signed-rank test, $+P<0.01$ (anosmic groups vs. control groups after the anosmic procedure), Mann-Whitney $U$ test. B, the BG curves before and after anosmic treatment in anosmic rats. Data are mean \pm SEM $(n=6), * p<0.05$ (after vs. before anosmic treatment), two-way repeated-measures ANOVA followed by Tukey-kramer test.
$\left.2+Y_{60}\right) \times 15 / 2+\left(Y_{60}+Y_{90} \times 2+Y_{120}\right) \times 30 / 2[$ Equation $1]$, where $Y_{x}$ represents the $B G$ level at time point $(x)$.

We applied the area of the BG curve to equation 1 and compared the AUC for the different conditions.

\section{Data analysis and statistics}

Data are presented as mean \pm SEM, unless otherwise indicated. The comparison of the latency to locate the cookie before and after the anosmic treatment in groups was used by Wilcoxon signed-rank test. The comparison of the latency between groups was assessed by Mann-Whitney $U$ test. The statistical significance of differences in BG curves between groups was assessed using two-way repeated-measures analysis of variance (ANOVA), followed by Tukey-kramer test. To determine the significance of differences in the area under the AUBGC in comparison with the control, Wilcoxon signed-rank test in Experiment 1 and one-way factorial ANOVA in Experiment 2-1 were performed, respectively. $P<0.05$ was considered significant.

\section{Results}

\section{Experiment 1: Effect of impaired olfaction on BG after glucose loading}

We showed that the anosmic treatment carried out by damaging olfactory cells with $\mathrm{ZnSO}_{4}$ solution applied into the nasal cavity was effective, since anosmic rats exhibited a significantly longer latency to locate the cookie hidden in the woodchips than the sham-treated control rats. As shown in Fig. 1A, there was a significant difference between the anosmic and control groups after the anosmic procedure $(P<0.01)$, and before and after treatment in the anosmic group $(P<0.05)$. Impairment of olfaction following intranasal infusion of $\mathrm{ZnSO}_{4}$ resulted in a marked downward shift $(P<0.05)$ in the BG curve, leading to a significant decrease $(P<0.05)$ in the AUBGC (Fig. 1B and Table 1).

\section{Experiment 2: Effect of addition of olfactory stimulus on BG after glucose loading}

As shown in Figure 2A and Table 1, sniffing of grapefruit odor for $15 \mathrm{~min}$ before glucose loading resulted in a marked changed the BG curve and significant decrease $(P<0.05)$ in the AUBGC compared with the 
control (odor-free air). By contrast, lavender odor induced no significant change in the BG curve (Fig. 2B and Table 1). Limonene, a major component of grapefruit oil, mimicked the effect of grapefruit odor (Fig. 2C). The BG curve for anosmic rats was not dependent on sniffing of the grapefruit odor, indicating that grapefruit odor affected the transmission of neural messages from the nasal cavity (Fig. 2D).

Table 1. Comparison of the area under the curve of blood glucose from starting to $120 \mathrm{~min}$ after the different glucose-loadings.

\begin{tabular}{clllll}
\hline \multirow{2}{*}{$\begin{array}{l}\text { AUBGC } \\
(\mathrm{mg} / \mathrm{dl} \times \mathrm{min})\end{array}$} & \multicolumn{2}{c}{ Experiment 1} & \multicolumn{2}{l}{ Experiment $2-1$} & \\
\cline { 2 - 6 } & Before & After & Control & Grapefruit & Lavender \\
\hline Mean \pm SE & $15127 \pm 261$ & $13787^{*} \pm 499$ & $15168 \pm 289$ & $14157 \dagger \pm 411$ & $15459 \pm 168$ \\
Median & 15234 & 13474 & 15002 & 14142 & 15355 \\
Range & 1669 & 2805 & 2094 & 2757 & 892 \\
\hline
\end{tabular}

${ }^{*} P<0.05$ (after vs. before anosmic treatment), Wilcoxon signed-rank test, $\mathrm{n}=6 ;+P<$ 0.05 (grapefruit vs. control), one-way factorial-ANOVA followed by Tukey-kramer test, $n=6$; AUBGC, area under the blood glucose curve.

\section{Discussion}

Physiologically, glucose metabolism is regulated primarily by various hormones, including insulin,
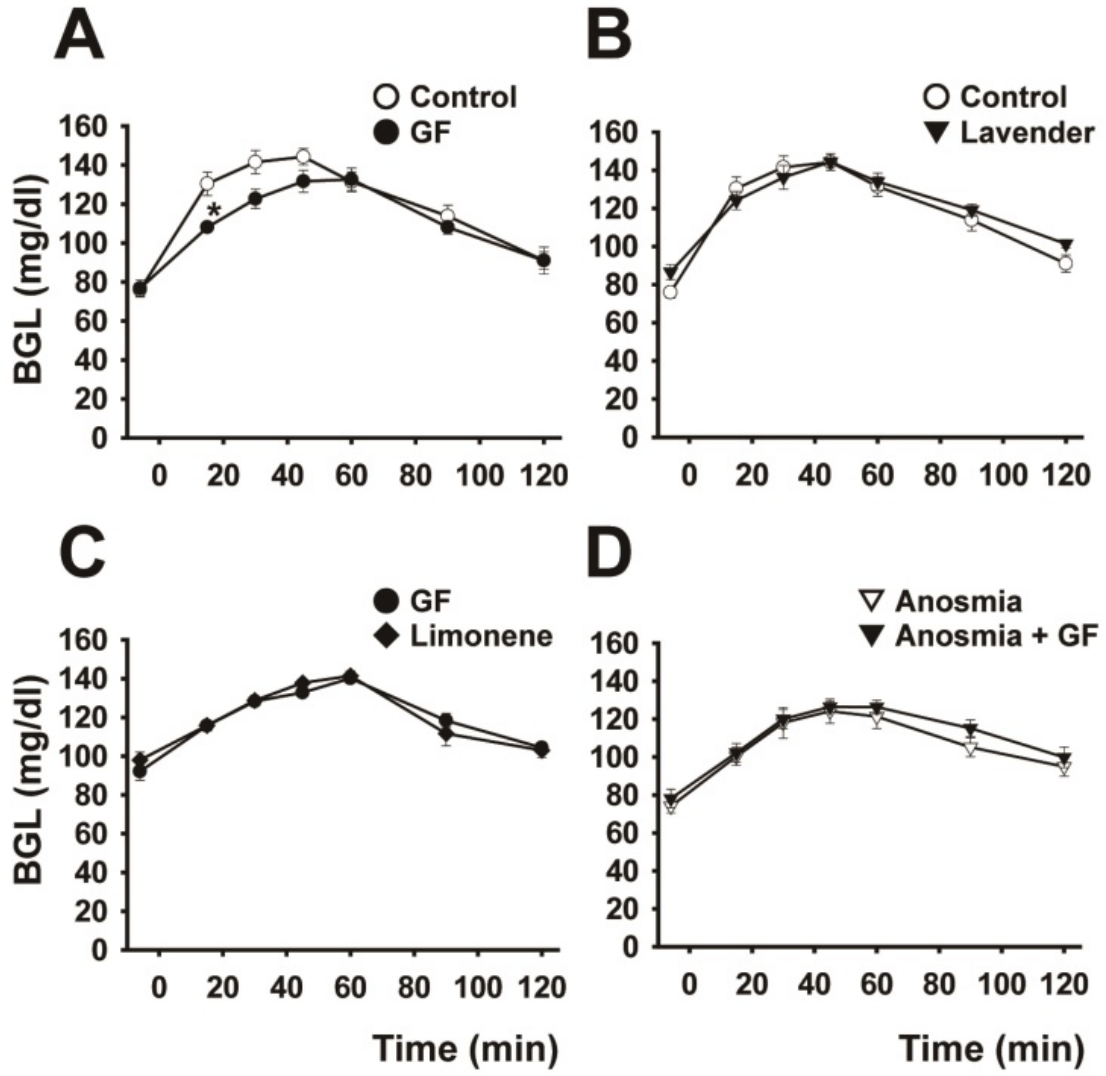

Figure 2. Effect of addition of olfactory stimulus on the blood glucose curve. The effect of odor stimulation on the blood glucose curve is shown under the following conditions: (A) control and sniffing of grapefruit odor for 15 min $(n=6)$, (B) control and sniffing of lavender odor for 15 min $(n=6),(C)$ sniffing grapefruit and limonene odors contained in grapefruit oil for $15 \min (n=5)$, and (D) sniffing grapefruit for 15 min before and after anosmic treatment $(n=6)$. Data are mean $\pm S E M$. $* P<0.05$, two-way repeated-measures ANOVA followed by Tukey-kramer test. glucagon, adrenaline, and noradrenaline [1]. Glucose ingestion during meals is the most efficient way to increase the BG level and is simultaneously modulated by chemical senses, such as olfaction and taste, which are strongly associated with food palatability via the ANS [10]. Especially, olfactory system is intimately linked with the endocrine system including digestive physiology [11]. For example, Lushchak OV et al. [12] demonstrated that food odors affect appetite, feeding and subsequent metabolic events such as insulin and glucagonlike signaling in Drosophila melanogaster. Acute exposure to vinegar odor triggers a rapid and transient increase in circulating glucose, and a rapid upregulation of genes encoding the glucagon-like hormone adipokinetic hormone, four insulin-like peptides (DILPs) and some target genes in peripheral tissues. However, the direct effects of these olfactory stimulation on BG levels after glucose ingestion remain to be elucidated. This is the first demonstration that chemosensory stimuli, particularly odor, modulates glucose ingestion-associated changes in BG levels in rats.

The most important finding of the present study is that experimental impairment of olfaction by intranasal infusion of $\mathrm{ZnSO}_{4}$ induces a marked downward shift in the BG curve. In our previous study [7], oral glucose loading in humans in conjunction with clamping the nose closed also produced a downward shift in the BG curve, together with increased salivary amylase (s-AMY) activity, which is a marker of sympathetic nervous activity [13]. One interpretation of the observed change in the BG curve under impaired olfaction conditions derives from the important role played by the olfactory system in anxiety reactions in both animals and humans [14]. We speculate that the temporary impairment of olfaction might have caused intense anxiety in the rats, with activation of the sympathetic nervous system, presumably because the anosmic rats were unable to process taste information [15].

Moreover, grapefruit odor (but not lavender odor) significantly altered the BG curve, despite stimulation prior to glucose loading. The effect of grapefruit odor could be explained by the 
ANS. That is, grapefruit odor increased and decreased the activity of the sympathetic and parasympathetic nerves, respectively, thus modulating digestion [6] and causing a downward shift in the BG curve.

Although it is plausible that the ANS plays a role in mediating ingestion-induced changes in BG level, we did not directly evaluate the activity of autonomic nerves. We are currently investigating the mechanism through which taste and olfactory inputs affect BG levels.

\section{Conclusion}

The present study showed that impairment of olfactory function following anosmic treatment lowers the BG curve in rats. Furthermore, olfactory stimulation before oral ingestion of glucose modulates ingestion-induced changes in BG levels, depending on the stimulus. The activation of sympathetic nerves by floral odors could be utilized as a strategy for controlling meal-induced changes in BG levels in humans.

\section{Acknowledgments}

This study was supported by JSPS KAKENHI Grant Number 23792338 and 25293409 from the Ministry of Education, Culture, Sports, Science, and Technology of Japan. We thank R. Komaki and H. Kunieda for gifted the essential oil.

\section{Author contribution}

T.T. and T.Y. designed the study and wrote and edited the manuscript. T.T. and S.B. acquired the data. T.T., S.T., and M.K. analyzed the collected data. All authors approved the final version of the manuscript. T.T. is the guarantor of this work and, as such, had full access to all data in the study and takes responsibility for the integrity of the data and the accuracy of the data analysis.

\section{Competing Interests}

The authors have declared that no competing interest exists.

\section{References}

1. Niijima A. Nervous regulation of metabolism. Prog Neurobiol. 1989; 33: 135-47.

2. Shepherd GM. Smell images and the flavour system in the human brain. Nature. 2006; 444: 316-21.

3. Abdallah L, Chabert M, Louis-Sylvestre J. Cephalic phase responses to sweet taste. Am J Clin Nutr. 1997; 65: 737-43.

4. Niijima A. Effects of taste stimulation on the efferent activity of the autonomic nerves in the rat. Brain Res Bull. 1991; 26: 165-7.

5. Inui-Yamamoto C, Furudono Y, Yamamoto T. Hedonics of taste influence the gastric emptying in rats. Physiol Behav. 2009; 96: 717-22.

6. Shen J, Niijima A, Tanida M, Horii Y, Maeda K, Nagai K. Olfactory stimulation with scent of grapefruit oil affects autonomic nerves, lipolysis and appetite in rats. Neurosci Lett. 2005; 380: 289-94.

7. Tsuji T, Tanaka S, Kida K, Bakhshishayan S, Kogo M, Yamamoto T. Disrupted normal ingestion during glucose intake modulates glucose kinetics in humans. Springerplus. 2015; 4: 621. doi: 10.1186/s40064-015-1419-3.
8. Kolunie JM, Stern JM. Maternal aggression in rats: effects of olfactory bulbectomy, $\mathrm{ZnSO}_{4}$-induced anosmia, and vomeronasal organ removal. Horm Behav. 1995; 29: 492-518.

9. Shen J, Niijima A, Tanida M, Horii Y, Nakamura T, Nagai K. Mechanism of changes induced in plasma glycerol by scent stimulation with grapefruit and lavender essential oils. Neurosci Lett. 2007; 416: 241-6.

10. Kitamura A, Torii K, Uneyama H, Niijima A. Role played by afferent signals from olfactory, gustatory and gastrointestinal sensors in regulation of autonomic nerve activity. Biol Pharm Bull. 2010; 33: 1778-82.

11. Palouzier-Paulignan B, Lacroix MC, Aimé P, Baly C, Caillol M, Congar P, Julliard AK, Tucker K, Fadool DA. Olfaction under metabolic influences. Chem Senses. 2012; 37: 769-97.

12. Lushchak OV, Carlsson MA, Nässel DR. Food odors trigger an endocrine response that affects food ingestion and metabolism. Cell Mol Life Sci. 2015; 72: 3143-55.

13. Yamaguchi M, Kanemori T, Kanemaru M, Takai N, Mizuno Y, Yoshida H. Performance evaluation of salivary amylase activity monitor. Biosens Bioelectron. 2004; 20: 491-7.

14. Clepce M, Reich K, Gossler A, Kornhuber J, Thuerauf N. Olfactory abnormalities in anxiety disorders. Neurosci Lett. 2012; 511: 43-6.

15. Uebayashi H, Hatanaka T, Kanemura F, Tonosaki K. Acute anosmia in the mouse: behavioral discrimination among the four basic taste substances. Physiol Behav. 2001; 72: 291-6. 